\title{
UN NUEVO ACERCAMIENTO AL ESTUDIO DE HERRAMIENTAS LÍTICAS DE LA CULTURA MILENARIA "LAS VEGAS" SANTA ELENA, ECUADOR.
}

\author{
A NEW APPROACH TO THE STUDY OF LITHIC TOOLS \\ OF ANCIENT LAS VEGAS CULTURE. SANTA ELENA ECUADOR
}

\author{
Yoshitaka Kanomata, Dr. \\ Tohoku University, Sendai, Japón \\ kanomata@m.tohoku.ac.jp \\ Andrei V. Tabarev, Dr. \\ Institute of Archaeology and Ethnography, Novosibirsk, Rusia \\ Julia V. Tabarev. Dra. \\ Institute of Archaeology and Ethnography, Novosibirsk, Rusia \\ Karen E. Stothert, Ph. D \\ Investigadora del MAAC, Guayaquil; Department of Anthropology, University of Texas \\ at San Antonio, Texas, EE.UU.
}

\section{RESUMEN}

La historia del estudio de la cultura Las Vegas ilustra un aspecto del proceso científico: se debe revisar repetidamente las interpretaciones de la evidencia arqueológica porque el desarrollo de técnicas de análisis más avanzadas nos conducen siempre a mejores interpretaciones del pasado. Se presenta unos ejemplos del progreso científico en el campo de la arqueología ecuatoriana y se enfoca en la tecnología lítica de la cultura Vegas conocida a base de evidencias arqueológicas recuperadas en sitios habitacionales ubicados en la Península de Santa Elena y ocupados originalmente en el Holoceno Temprano. Se presenta una descripción morfológica de herramientas líticas (lascas de piedra) manufacturadas por manos de la gente Vegas, pero se enfatiza un nuevo programa de observaciones sistemáticas microscópicas que ha resultado en inferir las funciones de algunas herramientas. La interpretación se basa en la identificación de huellas de uso (use wear) que aparecen bajo magnificación como estrías y pulimiento que son los resultados del uso del filo de las lascas en diversos trabajos. Se sugiere que una lasca servía para tallar hueso o asta de venado, mientras que otra se empleaba antiguamente en el raspado en seco del cuero de un animal. El próximo paso de la investigación es la replicación de unas lascas Vegas utilizando piedra natural de Santa Elena: luego con estas lascas se emprende actividades experimentales (como cortar y raspar en una variedad de fibras vegetales y tejidos de origen animal) para luego poder inferir las actividades específicas realizadas por los antiguos pobladores de la costa del Ecuador.

Palabras clave: Cultura Las Vegas, Tecnología lítica, Holoceno temprano

\begin{abstract}
The history of the study of the Las Vegas culture illustrates an aspect of the scientific process: it is advisable to review repeatedly the interpretation of archaeological evidence because the development of more advanced techniques of analysis result in better interpretations of the past. Several examples of scientific progress in Ecuadorian archaeology are presented, but this study focuses on the lithic technology of the Las Vegas culture known from evidence recovered from habitation sites located on the Santa Elena Peninsula that were occupied during the Early Holocene. We summarize the manufacture of Vegas tools based on their morphology, but we emphasize an innovative program of systematic microscopic observation that has resulted in the inference of the functions of ancient tools. This interpretation is based on the identification of patterns of use wear or damage (microscopic striations and zones of polish) that appear on the edges of the lithic flakes manufactured by Vegas people. It is suggested that one lithic flake functioned to work bone or deer antler, while another was used to process dry animal hide. The next step in this investigation is to replicate some Vegas flake tools using local stone material in Santa Elena. Then it will be necessary to undertake experimental activities (like cutting and scraping a variety of materials such as vegetable fibers and animal flesh and bones) in order to produce use wear patterns on the duplicate tools that correspond to known activities. By comparing the experimental tools
\end{abstract}


with ancient Vegas tools, it will be possible to infer more precisely the activities performed by the ancient inhabitants of the coast of Ecuador.

Keywords: Las Vegas Culture, lithic technology, Early Holocene

Recibido 18/12/2013

Aprobado 12/02/2014

\section{Introducción}

El presente texto tiene por motivo presentar un informe preliminar de una nueva investigación de artefactos de la cultura Las Vegas. Estas comunidades antiguas vivían en la Península de Santa Elena antes de la época de la producción y uso de vasijas de cerámica. La mayoría de los objetos recuperados en excavaciones arqueológicas en sitios de esta cultura son artefactos de piedra fabricados con varios materiales y con distintas técnicas resultando en herramientas de funciones y formas distintas. Afortunadamente, casi la totalidad del conjunto de artefactos líticos (de piedra) excavados y analizados entre 1977 y 1982 están todavía accesibles para investigadores capaces de tomar datos de la evidencia y llegar a nuevos conocimientos del pasado, y ahora hay nuevos acercamientos a la evidencia lítica de la cultura Las Vegas.

Stothert ha cumplido más de cuarenta años investigando la cultura Las Vegas (de los Amantes de Sumpa) y ha observado que la ciencia es un proceso largo y fascinante: durante todo este tiempo las interpretaciones arqueológicas se transformaban continuamente mientras que las evidencias se acumulaban y los métodos y teorías de la disciplina mejoraban. En los siguientes párrafos se cuenta la saga de la investigación multidisciplinaria que ha resultado en conocer los logros del pueblo Las Vegas.

En 1971, Stothert emprendió en Santa Elena la investigación de las tres presuntas culturas pleistocénicas definidas y publicadas en 1970 por Edward Lanning (1): estos se llamaron "Exacto, Manantial y Carolina". En breve, ella realizó excavaciones en todos los sitios de Lanning donde artefactos de piedra se encontraban en la superficie de la tierra, pero esta investigadora no pudo identificar artefactos debajo de la superficie de la tierra. Además, todos los presuntos sitios arqueológicos se ubicaron a lado de construcciones recientes o donde el horsteno ${ }^{i}$ aparecía en la superficie debido a la erosión de las formaciones geológicas. Todos los sitios lejos de los lugares de construcción moderna mostraron evidencias de que mineros modernos venían picando y triturando el horsteno para vender el cascajo a compañías de construcción para su uso en fabricar concreto. En el año
1971, fue posible ver lascas líticas no diferentes a las del complejo "Exacto" empotradas en la acera de cemento del Club de Ancón, a pocos metros de un pequeño arroyo (el Sitio 95 de Lanning), donde una carretilla de materiales de construcción y artefactos tipo "Exacto", habían sido descargados después de fundir el cemento de la acera.

Indagaciones comprobaron que durante por lo menos 20 años la compañía petrolera había comprado fragmentos de horsteno a los hombres picapedreros independientes de Santa Elena. Con el fin de asegurar que todos los artefactos conocidos fueron productos de los picapedreros actuales, Stothert invitó a un minero de Santa Elena a que redujera varias piedras grandes de horsteno recolectadas en diferentes afloramientos de piedra en la península. Se hizo la prueba sobre unas lonas, para que los productos pudieran estudiarse posteriormente. El picapedrero trabajó con mazos de fierro, rompiendo el horsteno en fragmentos sobre un pedregón que le servía de yunque, produciendo lascas aptas para la mezcla del concreto, y dejando también fragmentos de núcleos. El estudio de los restos de este trabajo, confirmó que los artefactos "tipo Exacto" fueron producidos al azar por un picapedrero de nuestros días.

Al no existir un sitio "Exacto" libre de la influencia de estos picapedreros, Stothert llegó a la conclusión que la evidencia no sostenía la hipótesis de la existencia de una industria prehistórica llamada "Exacto". Se puede leer una descripción del proceso científico que resultó en la derrota de todos los tres complejos culturales (Exacto, Manantial y Carolina), en el libro La Prehistoria Temprana de la Península de Santa Elena: Cultura Las Vegas (Stothert 2; 3, pp. 15-19). La ciencia tiene la habilidad de corregir los errores del pasado, y por medio de investigaciones creativas los científicos revelan interpretaciones novedosas y más confiables.

Pequeñas excavaciones realizadas en 1971, comprobaron que el sitio epónimo de la cultura Las Vegas satisface todos los requisitos arqueológicos para ser considerado un paradero humano ocupado en los albores del Holoceno. En aquel entonces el sitio fue situado afuera de la zona urbana de Santa Elena en una loma cerca del río Las Vegas (ahora en el campus del Museo de los 
Amantes de Sumpa). Reconociendo la importancia del Sitio 80, el Dr. Olaf Holm, Director Fundador del Museo Antropológico del Banco Central (Guayaquil), invitó a Stothert a llevar a cabo la investigación en el Sitio 80. Los resultados de la primera temporada de trabajo nos convencieron de la necesidad de proteger el sitio porque es (y sigue siendo), una fuente de evidencia importante. Los trabajos duraron hasta 1982, pero una buena parte del sitio queda sin excavar hasta el día de hoy. Ya que el proceso de excavación resulta en la destrucción de evidencia arqueológica, es aconsejable esperar hasta que los métodos y estrategias científicas de la arqueología se evolucionen más-para poder aprender más en el futuro.

Basándose en los resultados de las investigaciones realizadas entre 1977 y 1982, se sabe que el Sitio 80 fue un campamento de la cultura Las Vegas, y que fue ocupado entre 10.800 y 6.600 años antes del presente (a.p.).i" Después de la excavación, Stothert hizo el análisis morfológico de los artefactos líticos que representaban la mayoría de los objetos preservados en el basurero Vegas. La descripción detallada (y con muchas ilustraciones), apareció en el informe publicado (3:63-103).

Las herramientas de piedra, concha y hueso recuperadas en el Sitio 80, representan sólo parte del inventario material de la Cultura Las Vegas. Desafortunadamente, la mayor parte de la cultura material que una vez debe haber existido, fue elaborada de madera, caña, y otras fibras vegetales que desaparecieron por completo junto con otros materiales perecederos de origen animal (como cueros y plumas). Los objetos arqueológicos preservados no proporcionan evidencias del cambio cultural durante la ocupación larga del sitio porque las muestras de artefactos diagnósticos fueron muy pequeñas y los objetos sencillos y utilitarios encontrados en este sitio rara vez mostraron cambios de estilo. Sin embargo, Stothert propuso la existencia de dos períodos: Las Vegas Temprano y Las Vegas Tardío, que se distinguieron empleando otras clases de evidencias descritas en el informe final del trabajo (3:102-103). Por ejemplo, por medio del estudio de los moluscos recuperados en OGSE-80, fue evidente que la gente del período Las Vegas Temprano consumía mayormente la concha prieta (un molusco del manglar apreciado hasta nuestros días), mientras que la dieta durante la fase Tardío fue diferente (Stothert 3, pp.187-202).

Se espera que en el futuro hayan más estrategias arqueológicas para descubrir diferencias de tecnología o de adaptación entre los pobladores más tempranos de Santa Elena y sus descendientes miles de años después.
En el informe mencionado (Stothert 3) se presentaron los resultados de una gama de investigaciones. Douglas Ubelaker (4, pp.105-131) hizo el estudio de los esqueletos del Sitio 80 e informó que los individuos de la cultura Las Vegas eran sanos, bien alimentados, y que gozaban de una expectativa de vida más larga que la de los pueblos prehistóricos posteriores en el Ecuador. Stothert describió los patrones de entierro del pueblo Vegas, y llegó a un mejor entendimiento de la espiritualidad de ellos (5, pp.133-170).

El análisis de los restos fáunicos del basurero realizado por Thomas Chase (6,pp.171-178) resultó en una descripción del aprovechamiento de animales silvestres (como venado y aves), que abundaban en la península en los tiempos Vegas. Además, tenemos la sugerencia de Wing (7,pp.179-186) que el perro de monte (antes llamado Dusicyon sechurae, pero ahora conocido por Pseudalopex sechurae), tenía un papel importante en la vida de la gente Vegas: la evidencia es que los huesos de este cánido eran muy comunes en el basurero y que elementos óseos y dentales acompañaban a los muertos en algunas tumbas. Actualmente algunos especialistas están investigando (con nuevos estudios genéticos) la cuestión del estatus de los "perros silvestres" de América del Sur: ¿Fueron semi-domesticados? ¿Sirvieron de compañeros o de alimento para el pueblo precerámico?

Stothert presentó las escasas evidencias vegetales del Sitio 80 (3, pp.215-224). La verdad es que hace treinta años casi no existía procedimientos analíticos para documentar el papel de plantas en la vida de los antiguos moradores del Neotrópico, y pocos arqueólogos sospecharon que los cazadores-recolectores en América del Sur tenían jardines donde cultivaban especies vegetales en camino de domesticación. En los años de los setenta, el análisis de polen se había desarrollado bastante, pero el polen no se preservó en los suelos alcalinos del Sitio 80 . Novedoso en el mundo fue el estudio de los fitolitos, una especie de microfósil muy especial: fitolitos ("piedras de plantas"), son estructuras inorgánicas (de sílica) que se desarrollaron en las células de algunas plantas (por ejemplo, en las semillas y en la corteza de los zapallos). Afortunadamente la joven Dolores Piperno, iii experta en el análisis paleobotánico del Neotrópico, llegó al Ecuador para integrarse en la investigación Vegas. Piperno informó sobre los microfósiles en un capítulo titulado "Los fitolitos de las plantas del OGSE-80 y la evidencia del cultivo de maíz en el Ecuador," y describió no sólo una nueva técnica de análisis, sino ofreció una hipótesis novedosa y sorprendente: que la Gente Vegas cultivaba el maíz mil años antes del 
período Valdivia (8, pp. 203-214). La evidencia e interpretación de Piperno ha cambiado la historia ecuatoriana. Felizmente, su primer informe fue nada más que un preludio.

En una época algunos arqueólogos norteamericanos dudaban de los resultados de Piperno, pero ella siguió buscando fitolitos en sedimentos arqueológicos (en Santa Elena, Panamá, Méjico, etc.). Resultó que la tierra empotrada en las superficies de herramientas grandes de piedra procedentes del Sitio 80 , y en la placa dentobacteriana de los dientes humanos de la cultura Las Vegas, contiene fitolítos diagnósticos. Ahora la evidencia de Piperno está aceptada en todas partes, y la cultura Las Vegas es famosa en el mundo de arqueología por figurar entre las primeras que presentan evidencias de uso de plantas cultivadas. Ahora, sabemos que una lista larga de plantas económicas (que cultivamos hasta nuestros días), fueron derivadas de especies silvestres que existían en Sudamérica al final del Pleistoceno. Debemos gracias a los pueblos que existían en aquel tiempo, porque sus cultivadores manipularon las plantas y provocaron cambios genéticos que resultaron en especies cada vez más útiles para el ser humano.

En fin, la gente Vegas, residente en la costa de Santa Elena, iv figuraba entre los agricultores más tempranos de las Américas: cultivaron tubérculos como leren (o lleren, la Calathea allouia), y plantas con semillas como zapallo y mate (calabazo), entre 9.000 y 10.000 años antes del presente. Cuando Piperno presentó evidencia del cultivo de maíz (Zea mays), en el sitio precerámico de los Amantes de Sumpa en unos 7.000 años antes del presente, pocas personas la creyeron, pero ahora la evidencia y la interpretación son completamente aceptadas por especialistas. Es patente que este proceso científico ha durado años, involucrando ideas muy controvertidas, pero toda la comunidad de científicos debe participar en criticar y defender ideas, mientras que seguimos buscando, con mejores acercamientos teóricos y técnicos mejor, nuevas evidencias e interpretaciones para poder forjar descripciones confiables de lo que ocurrió en el pasado. Se puede leer los resultados del estudio de fitolitos de los contextos Vegas en español y en inglés $(8,9,10,11,12,13,14)$.

La investigación de los fitolitos de Vegas ha durado ahora más de 30 años y ha sido un gran éxito. Pensando en estos logros, Peter Stahl y Stothert tomaron la decisión de armar un nuevo estudio de todas las colecciones de huesos de animales y de moluscos de la cultura Las Vegas. Tres puntos son importantes: primero, que casi todos los restos excavados en sitios de la cultura Las
Vegas todavía existen en buenas condiciones o en una bodega en Santa Elena o en la reserva del Florida State Museum (donde trabajaban los colegas investigadores Wing y Chase, ya mencionados); segundo, el análisis original se basó en muestras pequeñas, dejando la mayor parte de la colección sin estudiar (originalmente por falta de fondos y tiempo); tercero, los métodos y técnicas para la identificación y análisis se habían mejorado mucho desde la fecha del estudio original en el año 1981. Además, hoy en día disponemos de modelos ecológicos más sofisticados que antes. Por estos motivos se decidió nuevamente analizar e interpretar el $100 \%$ de la colección fáunica con la ayuda de expertos en mamíferos (P. Stahl); peces (P. Béarez); aves, reptiles y anfibios (M. Tellkamp), y moluscos (K. Clark). Recibimos apoyo de la National Science Foundation (EE.UU.), y junto con la colaboración del Instituto Nacional de Patrimonio Cultural en Guayaquil, se realizó el trabajo de laboratorio.

Ahora, un grupo más amplio de autores está trabajando sus informes finales y preparando un nuevo libro bilingüe (español/inglés), de divulgación científica sobre la cultura Las Vegas. Hay numerosas sorpresas, entre las cuales están nuevas identificaciones de aves y moluscos que mejoran nuestra reconstrucción del medio ambiente en el período Vegas. La idea es escribir una historia cada vez más detallada, inclusiva y confiable.

\section{Desarrollo}

\section{Leyendo las Piedras: Resultados Preliminares del Estudio del Con- junto Lítico de la Cultura Precerá- mica Las Vegas, por Kanomata, Ta- barev y Tabarev}

Durante dos semanas, en el mes de septiembre de 2013, un equipo bi-nacional japonés-ruso, formado por tres arqueólogos (Kanomata, A. Tabarev y J. Tabarev), se enfocaba en el estudio de las colecciones Las Vegas guardadas en el Campamento Cautivo, La Libertad, Provincia de Santa Elena. Estos investigadores, especializados en las herramientas líticas del período Paleolítico de Japón, Siberia y Eur-Asia (15), tienen interés en todas las costas del Océano Pacifico, incluyendo la del Ecuador (16). Esperamos que esta primera experiencia en el Ecuador sea preludio a investigaciones más ambiciosas en cooperación con instituciones científicas del país.

La cultura Las Vegas es el complejo arqueológico más antiguo que se conoce en la costa del Ecuador (10.800 a 6.600 a.p.). La interpretación de la evidencia arqueológica indica un sistema econó- 
mico efectivo enfocado en parte en recursos marítimos, y por eso nos interesa realizar comparaciones con culturas del litoral de Asia.

Observaciones preliminares. Entre los desafíos prácticos y teóricos de nuestra investigación figuran, primero, algunos problemas de la terminología: hay palabras que se utilizan en el ruso y el japonés que no tienen equivalentes exactas en español. Además, hace falta una base experimental con materiales locales para poder interpretar los patrones específicos de uso en los filos de las herramientas ecuatorianas. Hay que señalar que las piedras recuperadas en los sitios Las Vegas representan solo una parte de la caja de herramientas de este grupo antiguo: seguro que ellos aprovecharon de implementos fabricados de materiales orgánicos, como bamboo, madera, concha y hueso, los cuales no se preservan en la tierra (Stothert 2,3,11,18).

Sin embargo, los siguientes puntos presentan nuestras observaciones preliminares sobre la industria lítica Las Vegas:

--Grupos de la cultura Las Vegas recolectaban material lítico natural para la confección de sus herramientas en la vecindad de los sitios habitacionales, específicamente en las quebradas y arroyos (estacionalmente secos), donde se encuentra hasta la fecha una gama de tipos de piedra incluyendo cuarcita (en forma de cantos rodados que servían antiguamente para martillar), horsteno (una piedra rica en sílice, llamada chert en inglés), calcedonia, jaspe, etc. Parece que el $90 \%$ de estas piedras se encuentran entre 5 y $10 \mathrm{~km}$ de los sitios habitacionales.

--Las personas que fabricaron las lascas con filo cortante (que hay en todos los sitios Las Vegas), utilizaron un canto rodado (de cuarcita) como martillo para golpear los trozos (o núcleos) de horsteno: utilizaron la percusión directa para desprender las lascas. Las lascas primarias tienen corteza por un lado del artefacto, mientras que las lascas secundarias no la tienen. Los artesanos Vegas no produjeron el tipo de lasca alargada y prismática que se conoce como blade en inglés o navaja. No se observa núcleos prismáticos tampoco. No es obvio que los picapedreros Vegas se preocuparon de eliminar la corteza sistemáticamente, antes de hacer lascas. En efecto, sin invertir mayor tiempo, destreza y energía en la producción de filo cortante, los grupos Las Vegas lograron satisfacer sus necesidades.

--En los sitios Vegas se recuperaron algunos artefactos que son pequeños núcleos casi agotados, los productos de la estrategia sencilla de percusión utilizada por los artesanos antiguos. Por su morphología estos artefactos podrían llamarse "micro-núcleos" (microcores), caracterizados por el desprendimiento de lascas pequeñas (reconocidas por su impresión negativa sobre la superficie del núcleo). En Asia y en el norte de las Américas, los "verdaderos" microcores son los resultados del desprendimiento de lascas por medio de presión (una técnica más sofisticada que resulta en lascas de forma más controlada), mientras que el método Vegas de desprender las lascas fue simplemente la percusión. Es posible que el número de micro-núcleos se aumentara en las colecciones Vegas, procedentes de los depósitos arqueológicos más tardíos (Período Las Vegas Tardío).

--La mayoría de las lascas Vegas fueron utilizadas sin modificación (o sea, quedaron en blanco, sin retoque después de su desprendimiento del núcleo). Sin embargo, cuando se enfoca en las lascas de horsteno o calcedonia de calidad superior (más vidrioso y sin fallas) se nota que estas fueron retocadas con más frecuencia y, a veces, convertidas en buriles (burinated, en inglés).

--No hay evidencia clara que demuestre el uso de técnicas bifaciales o unifaciales en la industria Las Vegas. Sin embargo, un fragmento que podría haber sido parte de una delgada herramienta bifacial fue recuperado de un contexto excavado con una fecha confiable de unos $10.000+300$ a.p. Este contexto corresponde al período Las Vegas Temprano cuando, tal vez, grupos Las Vegas fueron más móviles y atravesaban un territorio amplio con herramientas bifaciales. Se recuperó también, en un contexto temprano, un artefacto único con abruptos retoques unifaciales.

El análisis del año 2012. Nuestro análisis sistemático y funcional de una selección de lascas de piedra fue realizado utilizando una técnica de observación microscópica para identificar huellas de uso (microwear, en inglés): éste es el método desarrollado por Keely (19), investigador conocido por el estudio de desgastes en las herramientas bajo lentes de alta magnificación. Nosotros empleamos un microscopio metalúrgico Olympus $\mathrm{BH}$ para observar y clasificar las distintas clases de alteraciones que aparecen como patrones de "pulimento" en los filos de los artefactos (Figura 1). Las observaciones fueron realizadas bajo una magnificación entre 100x y 400x.

En total, se realizaron observaciones microscó- 
picas en 23 artefactos líticos del Sito 80 (el sitio epónimo de la cultura Las Vegas). Lamentablemente, se reconoció pulimento por azar en los filos de algunas lascas: estas huellas fueron producidas por el tamizado (en una criba de malla metálica), después de su excavación por arqueólogos. Este pulimento esconde cualquier huella de uso anterior.

Resultados significativos y planes para el futuro. Aunque la mayoría de las lascas Vegas no presentaron huellas reconocibles, tres lascas quebradas (snapped flake tools, en inglés) presentaron pulimento (relativamente obvio) causado por uso, con estrías señalando la dirección de movimiento del artefacto durante la faena, (Figura 1: (anexos) ver artefactos LV80-1, 2 y 3). Los filos fueron empleados para raspar o rayar en una dirección vertical. Se reconoció, en dos de estas lascas, un tipo de pulimento que se identificó con trabajos en hueso o asta de venado (Fotos 1, 2, $7,8)$ (anexos). La identificación se basa en nuestra experiencia con artefactos del Paleolítico de Asia y en experimentos en los cuales se replica el uso de artefactos con los materiales indicados. Además, otra lasca (de estas quebradas), tiene huellas que implican su uso en raspar cueros secos (Fotos 4 y 5) (anexos). En resumen, estas lascas quebradas fueron utilizadas principalmente en la faena de recursos animales.

Aunque los antiguos picapedreros Las Vegas no fabricaban puntas de proyectil de piedra y como no tenemos evidencia de las lanzas confeccionadas de madera o caña, todavía se puede imaginar que los hombres y mujeres de aquel entonces atraparon animales con puntas, trampas y redes fabricadas de materiales orgánicos. Con experimentos, involucrando la replicación y uso de herramientas antiguos, será posible identificar las huellas microscópicas en lascas líticas que resultaron de la faena de fibras vegetales, hace miles de años.

No hay rastro de obsidiana en las colecciones Las Vegas, hecho que debilita la idea de contacto o intercambio entre comunidades Vegas y grupos contemporáneos de la sierra ecuatoriana. Se tuvo la oportunidad de examinar puntas de proyectil y artefactos bifaciales (retocados en sus dos superficies opuestas), de sitios del complejo El Inga ubicados cerca de Quito, donde los arqueólogos han identificado unas fuentes del apreciado vidrio volcánico (obsidiana)—material que no llegaba a las manos de la gente Las Vegas. También revisamos artefactos del complejo Cubilán, material excavado por Temme $(20,21)$ en la sierra sur. Tomando en cuenta la antigüedad de Cubilán (10.300-9.200 antes del presente), es llamativa la idea de contacto entre los antiguos pobladores de Loja y los de la costa en el Holoceno Temprano, pero no se observó semejanzas tecnológicas, y por ahora faltan otras evidencias arqueológicas que sostengan la hipótesis de contactos.

El presente proyecto ha sido la primera oportunidad, como científicos del Japón y Rusia, de trabajar en Sudamérica. El estudio de las colecciones de los sitios Vegas se limitó a muestras pequeñas de artefactos, sin embargo la oportunidad de trabajar ha estimulado nuestro interés en continuar el análisis tecnológico y de patrones de uso de herramientas líticas en el Ecuador. Para el futuro, quisiéramos pensar en la posibilidad de investigar las industrias de otras culturas prehistóricas, posteriores a Las Vegas, cuando grupos produjeron artefactos líticos distintos y más refinados que los de los pobladores precerámicos de Santa Elena.

Aunque unos pocos objetos de fibra, cuero, madera, y hueso han sido recuperados de tumbas, basureros y conchales prehistóricos en el Ecuador, la mayoría de los artefactos fabricados de distintos materiales orgánicos (disponibles en estas tierras tropicales), han desaparecido del récord arqueológico. Sin embargo, en el futuro, con el análisis microscópico de herramientas líticas, será posible precisar los materiales trabajados con los implementos de piedra.

El primer paso en nuestra metodología es realizar estudios funcionales, o sea experimentos controlados en los cuales realizamos trabajos con herramientas líticas. En Santa Elena, por ejemplo, deseamos empezar con piedra local, y con este material fabricar herramientas similares (en tecnología y morfología), a los de las culturas antiguas. Luego hay que utilizar el artefacto en la ejecución de una variedad de tareas (cortar, raspar, rayar, perforar, serruchar, descuartizar, etc.), en materiales como cuero fresco, cuero seco, madera, hueso, concha, piedra, cerámica, fibras vegetalesmateriales presentes en el pasado. La función de la herramienta de piedra debe ser interpretada en conjunto con los materiales recién mencionados.

El próximo paso será comparar (bajo magnificación) las huellas de desgaste en los artefactos prehistóricos con los desgastes producidos en los experimentos actuales. Este proceso de inferir funciones antiguos tiene implicaciones por la identificación de actividades y de adaptaciones en los medioambientes tropicales. Una vez que hay mejores reconstrucciones de conjuntos líticos, (procedentes de contextos arqueológicos); habrá la posibilidad de desarrollar el concepto de "organizaciones tecnológicos." Para los arqueólogos, el estudio de huellas de uso (microwear), contribuye 
a la interpretación de la utilización de espacio en los paraderos antiguos, al entendimiento de los procesos de formación de sitios, y a la descripción de la estructura de los mismos.

\section{Conclusiones}

La metodología esbozada en la sección anterior, se ha desarrollado sólo en los últimos años, y ahora permite conocer no solo los detalles de la tecnología lítica, (tal como fueron talladas las herramientas), sino también las funciones específicas de herramientas individuales. Ahora existe la posibilidad de conocer mejor las actividades de los antiguos pobladores que utilizaron implementos de piedra, (aunque las huellas de uso son más visibles en la materia prima fina y vidriosa).

En el futuro será interesante comparar la tecnología y adaptación de los moradores precerámicos de la costa del Pacífico de las Américas con la de grupos contemporáneos, en la costa del Pacífico de Asia. Ahora hemos realizado el sueño de tomar el primer paso hacia el estudio más tecnificado de la lítica Vegas, pero quedamos con pocos datos sobre la evolución económica entre el período Vegas Temprano y el Tardío. En el futuro nos toca emprender un mejor acercamiento a los artefactos líticos que permitirá la descripción de las actividades típicas de las dos fases, y tal vez evidencia de cambio a través del tiempo.

Para el arqueólogo, el objetivo de las labores es desarrollar el conocimiento del modo de vivir de los grupos humanos que anteriormente poblaron la tierra. Además, la mayoría de los arqueólogos desea difundir los nuevos conocimientos derivados de sus investigaciones científicas: esto hacemos por medio de publicaciones científicas y populares; en libros escolares y académicos, en televisión y otros medios de comunicación, y especialmente en museos, que son recursos educativos que también fomentan orgullo e identidad en la comunidad (sin mencionar turismo). Un buen museo es el que tiene exposiciones basadas en investigaciones previas y que pone en valor el contenido de sus vitrinas y ambientaciones. Con las investigaciones aquí resumidas, se ha iniciado una nueva etapa en el estudio de la cultura Las Vegas.

\section{Agradecimientos}

Nuestra gratitud al personal del Instituto Nacional de Patrimonio Cultura (INPC), Dirección Regional Guayaquil, por su apoyo. K.S. agradece especialmente a Petroindustrial/La Libertad, filial de Petroecuador, que ha hecho posible investigaciones arqueológicas en Santa Elena desde hace muchos años. Y.K,, A.T, y J.T. agradecen a la Dra. Karen Stothert por esta oportunidad de trabajar con las colecciones Las Vegas y por una excelente introducción a la arqueología ecuatoriana.

'Esta es la piedra local utilizada para hacer herramientas líticas de filo cortante en el período prehistórico y para hacer construcción con cemento en el presente.

ii Estas son fechas determinadas por el método de radiocarbono. Hoy en día estas fechas tienen que calibrarse para que correspondan a nuestro calendario moderno. En términos calibrados, 10.800 a.p. corresponde a una fecha de calendario entre 13.820 y 11.350 a.p.; y 6.600 corresponde a una fecha entre 7820 y 7310 a.p. Todas las fechas en este artículo estan "sin calibrar."

iii Entre algunos amigos en Santa Elena la Dra. Piperno (ahora distinguida científica de la Institución Smithsoniana en Washington) se conoce por el apodo "Dolores Guasango" por haber sufrido un envenenamiento terrible en el año 1979 después de haber sacado una muestra botánica del árbol vengativo (un guasango). La ciencia demanda sacrificios en el camino hacia el conocimiento.

iv Es probable que grupos precerámicos asentados en la Cuenca del Río Guayas también cultivaban plantas en sus jardines hace 9000 años, pero falta recuperar la evidencia arqueológica (Stothert y Sánchez 16).

$\checkmark$ Este proyecto fue patrocinado por la "Russian Scientific Foundation for Humanities, "Grant \#1201-00001a, con título: "Ancient Cultures of Colombia and Ecuador"; y por la "Foundation for Grantsin-Aid for Scientific Research, Japan Society for the Promotion of Science" (No. 23720376).

\section{Referencias Citadas}

(1) Lanning, EP. Pleistocene man in South America. World Archaeology 1970; 2(1):90-111.

(2) Stothert KE. Review of the early preceramic complexes of the Santa Elena Peninsula, Ecuador. American Antiquity 1983; 48(1):122-127.

(3) Stothert KE. La prehistoria temprana de la península de Santa Elena, Ecuador Cultura Las Vegas. Con contribuciones de T. Chase, D. Piperno, D. Ubelaker, E. Wing. Miscelánea Antropológica Ecuatoriana. Serie Monográfica 1988; 10. Guayaquil: Museos del Banco Central del Ecuador.

(4) Ubelaker, D.H. 1988 Restos de esqueletos humanos del sitio OGSE-80. En Stothert 1988:105132.

(5) Stothert KE. En: Stothert KE, editor. La prehistoria temprana de la península de Santa Elena. 
Guayaquil. Miscelánea Antropológica Ecuatoriana, Serie Monográfica 10, 1988. Capítulo VI, Patrones de entierros. Museo del Banco Central del Ecuador 1988; 133-169.

(6) Chase, T. Restos fáunicos. En: Stothert KE, editor. La prehistoria temprana de la península de Santa Elena. Guayaquil: Miscelánea Antropológica Ecuatoriana, Serie Monográfica 10. Museos del Banco Central del Ecuador; 1988. Capítulo VII; p. 171-178.

(7) Wing, E. S. 1988 Dusicyon sechurae en contextos arqueológicos tempranos. En Stothert 1988:179-198).

(8) Piperno, DR. Primer informe sobre los fitolitos de las plantas del OGSE-80 y la evidencia del cultivo de maíz en el Ecuador. En: Stothert KE, editor. La prehistoria temprana de la península de Santa Elena. Guayaquil: Miscelánea Antropológica Ecuatoriana, Serie Monográfica 10. Museos del Banco Central del Ecuador; 1988. 1988:203213.

(9) Piperno D, Andres T, Stothert K. Phytoliths in Cucurbita and other Neotropical Cucurbitaceae and their Occurrence in early archaeological sites from the Lowland American Tropics. Journal of Archaeological Science; 2003; 27:193-208.

(10) Piperno D, Pearsall D. The origins of agriculture in the Lowland Neotropics. Academia Press, San Diego, California.

(11) Piperno DR, Stothert K. Phytolith evidence for Early Holocene Cucurbita domestication in southwest Ecuador. Science; 2003 feb 14; 299:10541057.

(12) Stothert, K.E. y D. Piperno, 2000 La cultura Las Vegas de los Amantes de Sumpa y el contexto del origen del cultivo de plantas domesticadas. Miscelánea Antropológica Ecuatoriana 9:51-72.

(13) Stothert, K.E., D.R. Piperno, y T. Andres, 2003 Terminal Pleistocene/Early Holocene human adaptations in coastal Ecuador: the Las Vegas evidence. Quaternary International 109111:23-43.

14) Stothert, K.E. y A. Sánchez Mosquera, 2012. Culturas del Pleistoceno Final y el Holoceno Temprano en el Ecuador. Tradiciones andinas tempranas: cultura, tecnología y medioambiente (P. Kaulicke y T. D. Dillehay, editores):81-120. Boletín de Arqueología PUCP 15. Lima, Perú

(15) Gunchinsuren B., Gladyshev S., Tabarev A.,
Kanomata Y., Khatsenovich A. Use-Wear Analysis on Palaeolithic Artifacts of Northern Mongolia. BuIletin of Tohoku University Museum 2013; 12:8-24.

(16) Tabarev, A.V. 2007 The South American Archaeology in the Russian Historiography. International Journal of South American Archaeology. N.1: 6-12.

(17) Stothert KE. The Preceramic Las Vegas Culture of Coastal Ecuador. American Antiquity 1985; 50: 613-637.

(18) Stothert KE. Trekking the Shore. Changing Coastlines and the Antiquity of Coastal Settlement. New York: Springer 2011. Capítulo Coastal Recourses and the Early Holocene Las Vegas Adaptation of Ecuador.; p.355-383.

(19) Keeley, LH. Experimental Determination of stone tool Uses. Chicago:University of Chicago Press; 1980.

(20) Temme, M. 1982 Excavaciones en el sitio precerámico de Cubilán, Ecuador. Miscelánea Antropológica Ecuatoriana 2:135-164.

(21) 2009 Cubilán. Dos Estaciones Precerámicas en el Curso Superior del Río San Felipe de Oña (Provincias: Loja y Azuay--Ecuador). Loja, Ecuador: Editorial Universidad Técnica Particular de Loja-Ecuador. 


\section{Figura 1 (ANEXOS)}

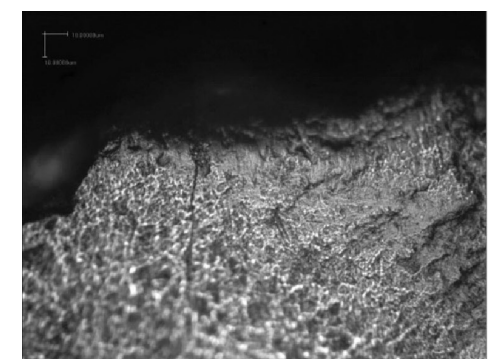

1. Pulimento tipo D1 con estrías verticales (x200)

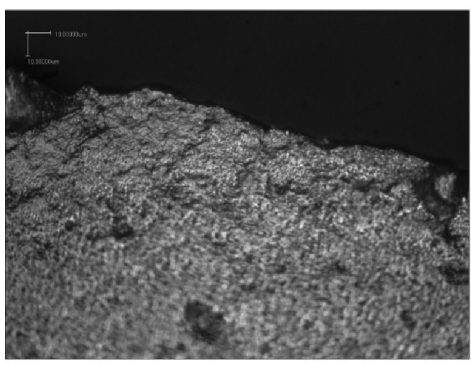

3. Filo sin uso (x200)

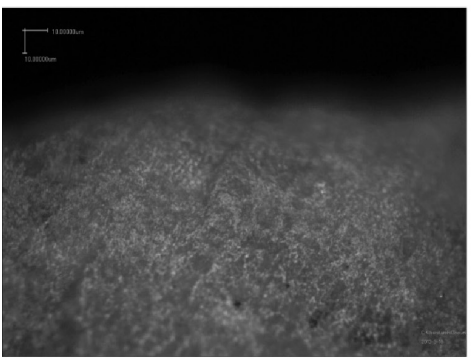

5. Pulimento tipo E2 con estrías verticales (x200)

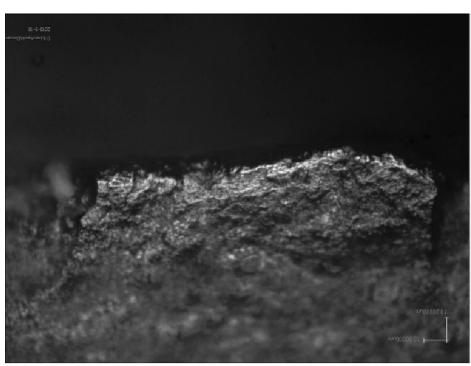

7. Pulimento tipo D1 con estrías verticales (x200)
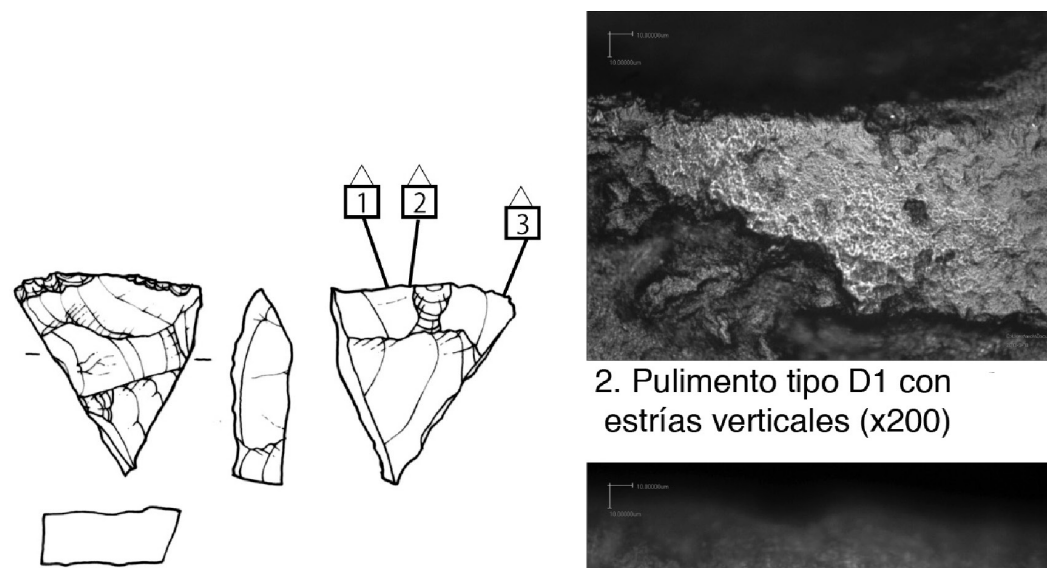

2. Pulimento tipo D1 con estrías verticales (x200)

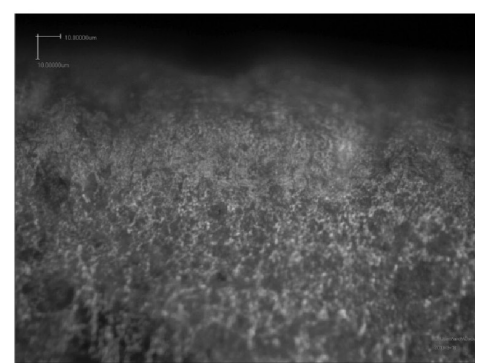

4. Pulimento tipo E2 con estrías verticales (x200)

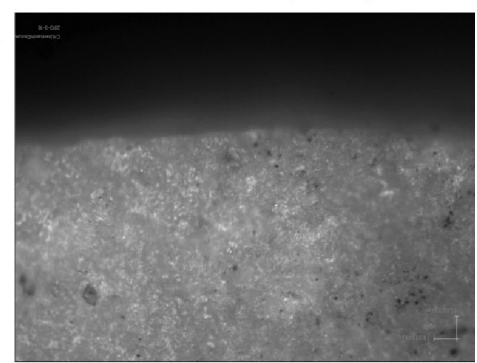

6. Filo sino uso (x200)
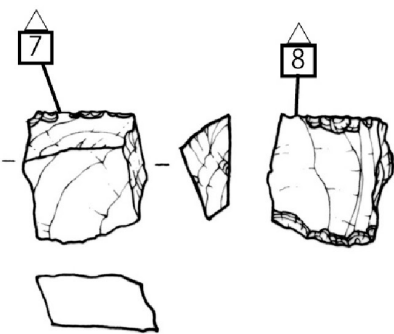

LV80-3

Escala $=75 \%$

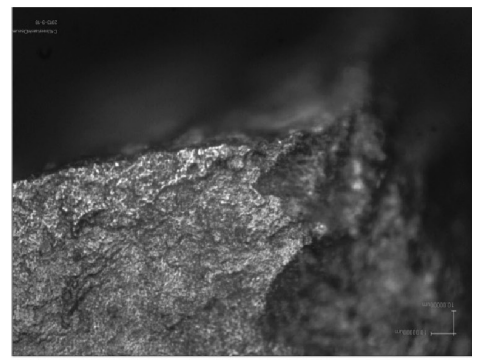
estrías verticales (x200)
8:Pulimento tipo D1 con

Figura 1. Huellas de uso identificadas en artefactos líticos (lascas de horsteno), de la cultura Las Vegas (Sitio 80). Los dibujos enseñan las dos caras y el corte transversal de tres lascas de piedra. Los números encuadrados indican la posición de cada huella que aparece en la foto adyacente. Observe la diferencia entre los filos utilizados y los filos sin uso. La magnificación es 200x. Pulimento del tipo D1 implica uso sobre hueso mientras que el tipo E2 sugiere uso sobre cuero seco. 